\title{
Mitochondrial COX4-1 deficiency leads to impaired nuclear DNA damage response resulting in proliferation deficits and premature senescence
}

\section{Liza Douiev}

Hadassah Medical Center

Chaya Miller

Hadassah Medical Center

Hadar Benyamini

Hebrew University of Jerusalem

\section{Bassam Abu-Libdeh}

Al-Makassed Islamic Charitable Society Hospital

Ann Saada ( $\square$ annsr@hadassah.org.il )

Hadassah Medical Center

\section{Research Article}

Keywords: COX, DNA damage, Fanconi anemia

Posted Date: November 4th, 2021

DOI: https://doi.org/10.21203/rs.3.rs-1026650/v1

License: (9) This work is licensed under a Creative Commons Attribution 4.0 International License.

Read Full License

Version of Record: A version of this preprint was published at International Journal of Molecular Sciences on April 8th, 2022. See the published version at https://doi.org/10.3390/ijms23084149. 


\section{Abstract}

Cytochrome- $c$-oxidase (COX), a multimeric protein complex, is the final electron acceptor in the mitochondrial electron transfer chain. Primary COX deficiency, caused by mutations in either mitochondrial DNA or nuclear-encoded genes, is a heterogenous group of mitochondrial diseases with a wide range of presentations ranging from fatal infantile to subtler. We previously reported a patient with primary COX deficiency due to a pathogenic variant in COX $4 / 1$ (encoding the common isoform of COX subunit 4), who presented with bone-marrow failure, genomic instability and short stature, mimicking Fanconi anemia (FA). In the present study, we demonstrated reduced proliferation and premature senescence in this patient's fibroblasts and in COX4-1 knockdown cells. Accumulative DNA damage coincided primarily with proliferative cells, indicating replicative stress. Expression analysis implicated DNA damage response which was verified by demonstrating impaired recovery from genotoxic insult and decreased DNA repair. Interestingly, our in-vitro findings recapitulate the patient's presentation and present status. Thus, we suggest that the premature senescence, resulting from accumulative DNA damage in COX4-1 deficiency is a protective mechanism to avoid malignant transformation in a similar manner to what was reported for FA and other "accelerated aging diseases".

\section{Introduction}

The mitochondrion is an intracellular cytosolic double membrane-bound organelle. It plays a critical role in many cellular processes, while one of its most crucial roles is the generation of energy as adenosine triphosphate (ATP) through the oxidative phosphorylation system (OXPHOS) localized in the inner mitochondrial membrane, is organized into five multimeric protein complexes $(\mathrm{Cl}-\mathrm{CV})$ and cytochrome $c$ and coenzyme Q. Electrons derived from nutrients are transferred via complexes I-IV (the electron transfer chain, ECT) while three of these are proton pumps, $(\mathrm{Cl}, \mathrm{CIII}$, and $\mathrm{CIV})$ generating an electrochemical gradient, which is utilized by CV (ATP synthase) to generate ATP. CIV (cytochrome coxidase, COX), is the terminal electron acceptor, catalyzing the reduction of molecular oxygen forming water. Mammalian COX is a dimeric composed of 14 subunits of which the largest three (COX I,II,III) are encoded by the mitochondrial genome while the remaining are nuclear-encoded. The synthesis and assembly of COX depends on numerous assembly proteins, while its activity is regulated by allosteric and posttranslational mechanisms, and by the expression of tissue-specific isoforms [1-3].

Primary COX deficiency belongs to a subgroup of mitochondrial diseases and up to 30 pathogenic variants related to human mitochondrial COX deficiency have been reported. The clinical manifestations of primary COX-deficiencies are heterogeneous, ranging from fatal infantile to subtler, mainly involving the brain and muscle, but frequently also heart, liver and pancreas. [4 -6]. Additionally, we and others have reported the occurrence of hematopoietic system involvement in patients with isolated COX deficiency [4-9]. In 2017 we reported a pathogenic variant (K101N) in the COX4/1 gene in a 3-year-old female patient suffering from poor growth, short stature, mild dysmorphic features, and bone marrow dysfunction. Pathogenicity was verified by complementation with the wild-type gene in the patient's fibroblasts [9]. 
COX4I1 is a nuclear gene encoding the common isoform of COX subunit 4 (COX4). COX4 has two isoforms; COX4 isoform 1 (COX4-1) and COX4 isoform 2 (COX4-2) encoded by the COX4/2 gene. COX4-1 is expressed under physiological conditions and is an essential structural and regulatory component of COX. COX4-2 the less common isoform, is primarily expressed in the lung and at lower levels in the placenta, heart, brain, and pancreas. COX4-2 is preferentially expressed under hypoxia and other stress conditions $[1-3,8]$. Recently we reported that isoform switch may act as a compensatory mechanism occurring in COX4-1-deficient cells (in both knockdown fibroblasts and in patient's cells). We demonstrated the involvement of HIF-1 a stabilization and nuclear translocation, that was followed by COX4 isoform switch under normoxic conditions. We thus hypothesized that the patient's relatively mild phenotype could be related to the isoform switch which preserving COX partial activity [10].

Our patient presented with clinical manifestations similar to Fanconi anemia. This syndrome is characterized by bone-marrow failure, genomic instability, and predisposition to cancer. The "classical" Fanconi anemia syndrome results from mutations in genes participating in the the FA pathway. This cellular process is activated when the DNA replication is blocked due to DNA damage. Activation of the FA pathway in intact cells, eventually, leads to DNA repair. Considering the patient's clinical phenotype, we also studied the contribution of COX deficiency to nuclear genomic stability and demonstrated the occurrence of elevated nuclear double-stranded breaks (DSB) foci in COX4-1-deficient cells, and also in COX6B1 deficiency. We found that the occurrence of these DSB foci was independent of ROS and ATP levels but depended on replicative state. We suggested that the patho-mechanism underlying COX deficiency, is associated with nuclear genomic instability, and is independent of oxidative stress or energy depletion [11-12]. Conversely, oxidative stress associated with mitochondrial dysfunction have both been previously implied in the classical form of Fanconi anemia, in patients' cells harboring mutations in the FA pathway genes [13-14]. Mitochondrial dysfunction also compels FA cells towards glycolytic metabolism [15] and this is similarly to our observations in the COX4-1-deficient cells [ 10].

To the best of our knowledge, the contribution of deficient mitochondrial respiration to genomic instability and its consequences has only been studied in the context of oxidative stress. In this study, we aimed to resolve the pathophysiological mechanism of COX4-1 deficiency with respect to its impact on the nuclear genomic instability and thereby on the potential clinical consequences. Our current study demonstrated that downregulated COX4-1 leads to premature senescence and impaired nuclear DNA damage response. This novel concept, could have implications for patient follow-up and treatment.

\section{Results}

Previously we showed that restrictive replication conditions (glucose-free and serum-free) lead to reduced levels of DSB in COX-deficient cells [12]. This evidence led us to hypothesize that COX4-1-deficient would reduce proliferation also in permissive conditions (High-glucose media) in order to circumvent replicative stress and DSB. We also evaluated DSBs under PARP inhibition and observed a major significant elevation in DSBs levels relative to untreated COX4-1-deficient cells, while the controls showed only a minor elevation. PARP inhibitors act by blocking the DNA repair pathways in which PARP participates 
(single-stranded break; base excision repair) resulting in the accumulation of DNA single-stranded breaks which subsequently leads to DSB. In cells with an intact DNA damage response (DDR), these breaks will be recovered through double-stranded repair pathways (homologous recombination; HR or nonhomologous DNA end joining; NHEJ). Accordingly, cells with impaired HR, for example those harboring pathogenic mutations in breast cancer genes $B R C A 1$ or $B R C A 2$, are hypersensitive to PARP inhibitors persistently accumulate DSB due to the error prone NHEJ. [16]. Since we previously showed that COX4-1-deficient cells are hyper-sensitive to PARP inhibitor treatment, we assumed that the HR mechanism in COX4-1-deficiency could be affected as well.

Consequently, we set out to examine proliferation and DDR in patient's cells harboring the K101N mutation and in COX4/1 knocked-down cells.

Decreased population doubling rate can be explained by either continuous cell cycle arrest (such as senescence) or cell death. Senescence is thought to be a tumor suppressive mechanism and an underlying cause of aging. In this process the cells remain viable, but do not proliferate. Senescent cells display a variety of cellular characteristics, including telomere attrition and senescence-associated expression of $\beta$-galactosidase (SA- $\beta$-Gal) activity [17]. To determine the percentage of both COX4-1deficient cells (HFF-shCOX4I1 and patient) and corresponding control cells for positive senescence marker, we stained for SA- $\beta$-Gal activity in three sequential passages of each cell line (Fig.2). We confirmed that both COX4-1-deficient cells (HFF-shCOX4I1 and patient; 78-84\% and 72-90\%, respectively) are more likely for being SA- $\beta$-Gal-positive than the corresponding controls (HFF-CV and healthy control; .(and $34-47 \%$, respectively $36-37 \%$

\subsection{Decreased proliferation and early entrance to cellular senescence without evidence of increase apoptosis}

To examine proliferation, we compared growth and viability of both COX4-1-deficient cells (patient fibroblasts and knockdown cells; HFF-shCOX4I1) with corresponding controls under permissive conditions (high-glucose medium) (Fig.1). To differentiate between proliferative and non-proliferative cells, we stained the cells, 72 hours after seeding equal amounts, against Ki-67, a well-established nuclear proliferation marker and with NucBlue which stains all nuclei irrespectively of replicative status. The cells were examined by fluorescence microscopy (Fig.1A) and distribution of each population and percent of Ki-67-positive nuclei out of total nuclei was determined and depicted in pie chats (Fig. 1B). We observed that the fraction of Ki-67-positive nuclei in both COX4-1-deficient cells (HFF-shCOX4I1 and patient) was significantly decreased relative to corresponding controls ( $30 \%$ and $50 \%$, respectively). This result was corroborated by trypan blue viability staining which displayed a reduced population doubling rate of both cells, compared to corresponding controls ( $49 \%$ and $59 \%$, respectively).

In addition, to verify our findings, we estimated the averaged telomeric lengths of both HFF-shCOX4I1 and HFF-CV, because as mentioned above, telomere attrition is additional marker for senescence.

Supplementary figure S1A represents the averaged telomeric length of knock-down cells relative to the that of the control cells (same nuclear background and passage). We observed, a marked ( $2 \mathrm{~Kb})$ 
reduction in the average telomeric lengths of HFF-shCOX4I1 cells relative to HFF-CV. We simultaneously assayed mitochondrial DNA content but found no difference between the cells (results not shown). This could possibly be attributed to upregulation of mitochondrial content in COX deficiency as we have previously reported [18]. To differentiate between senescence and apoptosis, we also performed TUNEL assay, but detected no significant difference, between both COX4-1-deficient cells and their respective controls (Supplementary Table 1). Notably, we did not detect any obvious difference in the occurrence of apoptotic nuclei when examining nuclei under the microscope in other experiments.

Altogether, our results suggest that the proliferation deficits in COX4-1-deficient cells is more likely attributed to senescence than apoptosis. This is also in accord with our previous observations [10] detecting increased glycolysis in these cells. Notably, metabolic shift, towards glycolysis occurs in senescence [19]

\subsection{Nuclear DNA instability coincides with proliferation}

We previously showed that COX4-1-deficient cells display high levels of DSB relative to controls by performing both phospho-histone H2AX Ser139 ( $\mathrm{YH} 2 \mathrm{AX}$ ) foci staining and neutral comet assay $[11,12]$. We also noted that cells maintained in glucose free- restrictive medium (where cells with impaired OXPHOS cease to proliferate) lacked DSB [12]. In view of our recent results (above) we speculated that senescence is a protective response to proliferative/ genotoxic stress, and therefore set out to determine whether elevated DSB are correlated with proliferative status in COX4-1 deficiency, by co-staining with both $\mathrm{yH} 2 \mathrm{AX}$ and $\mathrm{Ki}-67$ (Fig.3 A). Although a positive co-staining of both markers was observed in many cells, COX4-1-deficient cells displayed a larger portion of co-staining.

The pie charts (Figure 3B-C) represent the distribution of both proliferative (Ki-67 positive) and nonproliferative (Ki-67 negative) cell population stained for DSBs ( $\mathrm{\gamma H} 2 \mathrm{AX}$-positive or negative). We observed a statistically significant $(\mathrm{p}<0.05)$ positive correlation between the fraction of proliferating Ki-67 HFFshCOX4I1 and patient's cells that were also $\mathrm{YH} 2 \mathrm{AX}$-positive (84\% and $82 \%$, respectively), while the proliferative fraction of control cells had a lower tendency ( 50\% yH2AXpositive) (Fig.3B). Vice versa, as expected the non- proliferative fraction disclosed a decreased occurrence of DSBs in all cells (Fig.3C). The difference of $\mathrm{YH} 2 \mathrm{AX}$ positive between proliferative and non-proliferative cells was most marked (82\% vs $48 \%$ ) in the patients' cells, supporting the concept that non-proliferation/ senescence status is protective mechanism.

\subsection{CEL-Seq2 analysis identified DNA repair as a top downregulated pathway in COX4-1 knockdown cells}

Our previous and present results show that COX4-1-deficient cells display high levels of DSB foci, which are correlated with their proliferative status and hypothesize that the observes senescence an attempt to ameliorate DNA damage due to replicative stress, possibly due to impaired DNA damage response (DDR) 
[11]. To explore this possibility, we performed CEL-Seq2 analysis of HFF-shCOX4I1 and compared the RNA expression pattern with the HFF control (with the same genetic background. Downregulation and enrichment in several genes linked to DNA repair was observed (the top 20\% downregulated genes are listed in supplementary material table 2, and depicted in Fig.4 A-B). The CEL-Seq2 results were verified by RT-qPCR in both HFF-shCOX4I1, patient cells and their respective controls examining five of the downregulated genes present in the DNA repair enrichment gene set (XPC, PCNA, GMPR2, IMPDH2, UPF3) in both COX4-1-deficient cells (Fig.4C). These genes are marked in the volcano plot; Figure 4B. We also detected a qualitative, but not quantitative correlation between HFF-shCOX4I1 and the patients' cells (Fig.4C). This can be attributed to different nuclear backgrounds. Nevertheless, a decreased expression in all five genes quantified observed in agreement with the CEL-Seq2 analysis.

Total RNA was isolated from (human foreskin fibroblast cell line (HFF) HFF transfected with a control vector (HFF-CV) or HFF with downregulated COX4I1 (HFF-shCOX4i1). A HiSeq assay CEL-Seq2 analysis was performed and analyzed with the DESeq2 package. Significance threshold was set as FDR $<0.1$. Downregulation of DNA repair detected by gene set enrichment analysis (GSEA). NES: normalized enrichment signal; FDR: false discovery rate (A). In the volcano plot, each dot represents a gene (B). The $x$-axis indicates the log2 (fold change) of the expression of HFF-shCOX4I1 relative to healthy control fibroblasts, and the $y$-axis reflects - $\log 10$ of the FDR-adjusted $p$-value of this comparison. The colored dots pass the threshold for FDR. Selected DNA repair genes in the volcano plot (XPC, PCNA, GMPR2, IMPDH2, UPF3) were validated by RT-qPCR in both COX4-1-deficient cells (HFF-shCOX4i1 and patient) with their corresponding controls. Values of RT-qPCR validation are presented as the log2 (fold change) in \pm SD of biological triplicates, ${ }^{\star} p<0.05$; ${ }^{\star \star} p<0.01$ (C).

\subsection{Impaired DNA damage response in COX4-1-deficient cells}

The present expression analysis, well as our previous results showing increased sensitivity to PARP inhibitor [12], led us to believe that the nuclear DNA repair system and specifically homologous recombination (HR) is less effective in COX4-1 deficiency. To address this question, we examined the ability of COX4-1-deficient cells (both HFF-shCOX4I1 and patient's cells) to recover from an external DNA insult, i.e., their ability to repair DNA damage that caused by a short exposure to DNA damaging chemical (Etoposide; EP). To that end we quantified $\mathrm{YH} 2 \mathrm{AX}$ foci at two different time points, after a 1 hour exposure to EP. As shown in Figure 5, both HFF-shCOX4I1 (Figure 4A, C) and patient cells (Figure 4B,D) display impaired ability to respond and fully-recover from the genotoxic insult, while in the controls, the damage was almost fully-repaired after 24 hours of recovery. Moreover, exposure to EP treatment induced a priori increase in YH2AX foci after 2 hours of "recovery". After 24 hours of recovery, both HFF-shCOX4I1 and patient cells displayed a significant prolongation of $\mathrm{YH} 2 \mathrm{AX}$ foci signal, while both control cells had almost comparable levels relative to untreated cells. This result indicates that the increased levels of DSB observed in COX4-1-deficient cells are most probably attributed to an impaired DNA damage response. 
In an attempt to further elucidate the mechanism, we specifically examine HR we employed a commercially available homologous recombination assay, which requires the co-transformation of cells with two plasmids encoding the LacZa gene but with two different mutations. Only a functioning HR is able to restore the intact gene. As transformation is much less efficient in primary cells such as HFF, we constitutively knocked-down COX4I1 in HEK 293 cell line (HEK293-shCOX4I1) instead. Knockdown efficiency, as well as the presence of DSB were both validated in the HEK293 cells before commencing, and the results were comparable to those we reported in HFF-shCOX4I1 cells [10] (supplementary figure S2). Subsequently, we co-transfected the mutant LacZa plasmids (dl-1\&dl-2) into both HEK293-shCOX4I1 and HEK293-CV, overnight and analyzed the resulting products by PCR (Fig.6) and detected $28 \%$ significant decrease in HR efficiency compared to control. Although the decrease is moderate, it was statistically significant and thus in accord with impaired DDR.

\section{Discussion}

Senescence or cell death are two different mechanisms aimed to cope with prolonged and persistent stress conditions or due to DNA damage response deficits to preserve genome integrity [20]. Premature senescence is a well-known phenomenon, that is related to an induction of senescence occurring at earlier stage of population doublings than the maximal number of passages that are usually observed in healthy cells [17]. An early entrance into senescence can be induced by several factors, including persistent DNA damage, elevated oxidative stress, or oncogene activation. COX4-1-deficient cells display significantly reduced proliferation (Fig.1). We hypothesized that the reduction in their ability to proliferate and could be related to premature senescence, that occurs in response to the persistent nuclear DNA damage that we have previously reported $[11,12]$. To test this issue, we stained SA- $\beta-G$ al in both COX4-1deficient and control cells, at three sequential passages. COX4-1-deficient cells indeed displayed significant high levels of SA- $\beta$-Gal staining (Fig.2) while ruling out cell death by apoptosis (supplementary table 1), in accord with premature senescence. Similar to our observations, FA patients' cells demonstrated cellular features related to senescence, including decreased proliferation, a short lifespan of fibroblasts and increased expression of SA- $\beta$-gal $[21,22]$. Telomere shortening is another feature of senescence and DNA breakage and is also associated with FA, which fits well with our present findings. Although the telomere shorting in our HFF-shCOX4I1 in vitro cell system was less prominent (Fig.S1A) than in blood cells from common FA complementation groups, the similarity is evident [23]. Indeed, recently, FA has been re-defined as an accelerated aging (AA) diseases (including premature aging syndromes and aging-associated diseases) [24]. Observing the clinical and cellular manifestations, it seems that COX4-1 deficiency could belong to this group as well.

In accordance with our current observations, another feature of AA is replicative stress. We demonstrated that COX4-1 deficiency coincides with accumulative DNA damage, primarily in Ki-67 positive cells (Fig.3). This is also compatible with FA pathway deficits which are characterized by accumulative DNA damage, replication crisis and chromosomal aberrations due to impaired to DNA damage response (DDR) [22]. To strengthen this hypothesis, we proceeded to examine DDR by expression analysis (Fig.4), and experimentally demonstrated that COX4-1-deficient cells indeed displayed an impaired ability to respond 
and recover from a short exposure to a genotoxic agent and together with a reduction in HR efficiency (Figs. 5,6). Notably, the present data are compatible to our previous results showing the patient's fibroblasts are cells hyper-sensitive to PARP inhibitor [12]. As noted above, this line of in vitro investigation was initiated by the phenotype that our patient initially presented at the age of $2 \mathrm{y}$ and the COX $4 / 1$ mutation was identified and reported when she was 5y (9). Recent evaluation at the age of 9y showed that she still presents with severe growth faltering (failure to thrive: weight of $12 \mathrm{~kg}$ (significantly less than -2 SD); height $80 \mathrm{~cm}$ (significantly less than -2 SD) and occipitofrontal circumference of $49 \mathrm{~cm}$ (just at - 2SD).) Nevertheless, she has reasonable school performance and adequate mental and psychosocial development. To the best of our knowledge, she did not develop any seizure disorder or any other abnormal neurological signs or symptoms including malignancy. Her last blood counts showed mild normocytic anemia with normal WBC and Platelets counts [Hb=11.3 gm. /dl; Hct=32.8\%; MCV=87.2 $\left.\mathrm{FL} ; \mathrm{BC}=3.76 \times 10^{\wedge} 6 / \mu \mathrm{l} ; \mathrm{WBC}=7.4 \times 10^{\wedge} 3 / \mu \mathrm{l} ; \mathrm{PLT}=438 \times 10^{\wedge} 3 / \mu \mathrm{l}\right]$. She is maintained only on a daily dose of $100 \mathrm{mg}$ vitamin $\mathrm{C}$ (that was initiated based on our previous studies in fibroblasts (25). Interestingly our in vitro findings recapitulate the stunted growth, in the presence of chromosomal instability but without evidence of malignant transformation in the patient.

Our in vitro study in cells has several limitations; First, we could not exclude the contribution of different nuclear and mitochondrial genomic backgrounds while examining the patient's fibroblasts and moreover age matched healthy controls were (for obvious reasons) not available. Still, generating the knock-down vs control from the same cell line enabled us to overcome this issue. However, since the HFFshCOX4I 1 cells are prematurely senescent the number of passages was very limited. Secondly, HEK293 were used for the HR assay since we were unsuccessful in co-transfecting these primary cell lines two plasmids. Moreover, this assay system does not have any means of simultaneous tracking of transformation efficiency (from our previous experience with fluorescence expression of one plasmid it is $\sim 70 \%$ ) thus the only mildly decreased HR efficiency observed could be due to less efficient transformation with two plasmids. Third; in this study we did not provide a direct link between impaired DDR and the COX. This issue will be addressed in the future.

To summarize, in the present study, we demonstrated in both COX4-1-deficient cells (knockdown cell line and patient's fibroblasts) a reduced proliferation rate that was not attributed to apoptosis but to premature senescence. We also showed that the accumulative DNA damage coincides primarily with proliferative cells, indicating replicative stress. Expression analysis implicated DNA damage response which we verified by demonstrating impaired recovery from genotoxic insult and decreased homologous repair. Interestingly our in-vitro findings recapitulate the patient's presentation and present status. Thus, we suggest that the premature senescence, resulting from accumulative DNA damage in COX4-1 
deficiency is a protective mechanism to avoid malignant transformation in a similar manner to what was reported for FA and other "accelerated aging diseases".

\section{Materials And Methods}

\subsection{Tissue cultures}

Previously established skin primary fibroblasts cultures from the patient (Informed consent was obtained IRB \#0485-09) (7), human foreskin fibroblasts (HFF-1) (ATCC, Manassas, Virginia USA) and HEK293 (Invitrogen, Carlsbad CA, USA) cell lines were maintained in high-glucose DMEM supplemented with $15 \%$ fetal bovine serum, L-glutamine, pyruvate, and $50 \mu \mathrm{g} / \mathrm{mL}$ uridine (Biological Industries, Beit Ha'emek, Israel). For immunocytochemistry, cells were seeded on u-slide 8 well-ibiTreat sterile tissue culture slides (NBT; New Biotechnology Ltd., Jerusalem, Israel). For RNA analysis, cells were grown in 6-well plates. For DSBs staining or proliferation assay, cells were seeded on u-slide 8 well-ibi-Treat sterile tissue culture slides. All cells were incubated at $37^{\circ} \mathrm{C}$ in a humidified $5 \% \mathrm{CO}_{2}$ atmosphere. Cells from passages 7 or less were used for the experiments. Counting and viability were determined by trypan blue staining.

\subsection{RNA interference}

We employed the MISSION® shRNA plasmid DNA vector system shRNA \#TRCN0000232554 to constitutively knockdown the expression of COX4/1 as we have previously described (7). A nonmammalian shRNA Control Plasmid DNA target served as a control vector (Sigma-Aldrich-Merck, Darmstadt, Germany). Briefly we introduced each of the DNA plasmids into HFF-1 or HEK293 cells by cotransfection with pLP1, pLP2, and pLP/VSVG plasmids using lipofectamine (ViraPower; Invitrogen, California, USA). Human foreskin fibroblasts and HEK293 cells were infected with viral supernatant containing polybrene. Stably transfected cells were selected with puromycin $(2 \mu \mathrm{g} / \mathrm{mL})$ for three-weeks. For experiments the cells were maintained in permissive medium without puromycin. Knockdown was verified by RT-qPCR as we have previously described [10].

\subsection{Immunofluorescence staining}

The cells were seeded on u-slide 8 well-ibi-Treat sterile tissue culture slides (ibidi GmbH Gräfelfing, Germany). On the following day, the cells were fixed with $4 \%$ Formaldehyde for 10 min at room 
temperature, and then permeabilized with ice-cold $90 \%$ methanol for additional 10 min at $4^{\circ} \mathrm{C}$. After blocking with $1 \%$ BSA/PBS for 30 minutes, at room temperature, slides were incubated with primary antibody for 1 hour, at room temperature. The cells were washed five times with PBS containing $0.05 \%$ Tween-20, and then were incubated with secondary antibody for 1 hour, at room temperature in dark. The following primary antibodies were used for immunofluorescence: Ki-67 (1:250; Cat\#: ab16667, Abcam, Cambridge, UK).

Secondary antibodies: anti-Rabbit Cy5 (Cat\#: 711-175-152) and anti-mouse DyLight 488 (Cat\#: 115-485062) (both from Jackson Immuno research, Laboratories, Baltimore Pike, PA, USA). The slides were subsequently washed five times with PBS and nuclei were stained with Hoechst 33342, NucBlue live cell stain (Molecular probes, Life technologies Eugene OR, USA). The cells were examined by fluorescence confocal microscopy, X40 (for analysis) magnifications (Nikon A1R). Image analyses were performed by the quantification of $\mathrm{YH} 2 \mathrm{AX}$ foci per nucleus using the Image $\mathrm{J}$ software http://imagej.nih.gov/ij (National Institute of Health, Bethesda, MD, USA).

\subsection{Senescence and Apoptosis Detection}

Senescence-associated $\beta$-galactosidase (SA- $\beta$-gal) staining was performed using the SenescenceGalactosidase Staining Kit (MBL International Crop., cat \#JM-K320-250) according to the manufacturer's protocol. The same amount (15000) of cells from the same passage were seeded on u-slide 8 well-ibiTreat slides. Following 48 hours, cells were washed with PBS X1 and fixed with the supplied fixing solution for 10 min at RT. Next, the cells were washed once in PBS to remove the fixing solution and incubated with a freshly prepared staining solution mix containing the X-gal substrate at $37^{\circ} \mathrm{C}$ overnight. Afterward, SA- $\beta$-gal-positive cells (senescent cells) were identified as blue-stained cells under X20 magnification using Nikon-TI fluorescence microscope and quantified with the ImageJ analysis software.

Apoptosis was estimated by Terminal deoxynucleotidyl transferase (TdT)-mediated deoxyuridine triphosphate (dUTP) nick end labeling (TUNEL) using the in-situ cell death assay kit (Roche Diagnostics $\mathrm{GmbH}$, Mannheim, Germany) according to the manufacturer's instructions. The nuclei of the apoptotic cells containing DNA strand breaks were stained green (TUNEL apoptosis signal) and the overall nuclei were stained with Hoechst 33342, and examined by fluorescence confocal microscopy, X40 (for analysis) magnifications (Nikon A1R). Image analyses were performed by ImageJ software where we analyzed the green fluorescence signals (TUNEL-apoptosis) per nucleus. Results are represented as the ratio between green (TUNEL signal) and blue (nucleus) intensities.

\subsection{Telomere length}


Cells were seeded in triplicated in a 6-well plate. and total genomic DNA was isolated 24 hours later using DNeasy ${ }^{\circledR}$ Blood\&Tissue Kit (catalog No. 69504 \&69506; Qiagen) and telomeric lengths were quantified by RTqPCR (Absolute Human Telomere Length and mitochondrial copy number dual quantification qPCR Assay Kit ScienCell, Carlsbad, CA, USA; Cat\# 8958) according to the manufacturer's instructions. The results were compared to reference genomic DNA containing a 100-base pair (bp) telomere sequence and average telomere length was calculated following the manufacturer's instructions.

\subsection{Nuclear DNA double-stranded breaks (DSB)}

Nuclear DSB were evaluated by staining cells grown u-slide 8 well-ibi-Treat sterile tissue culture slides with antibodies against phospho histone $\mathbb{}^{\mathrm{H}} 2 \mathrm{AX}$ Ser139 using the Oxiselect DNA Double Stranded Break Staining Kit according to the manufacturers' instructions (Cell Biolabs Inc., San-Diego, CA, USA). For DNA damage recovery experiments, the cells were incubated with a $100 \mu \mathrm{M}$ of Etoposide (EP) for 1 hour, subsequently, the medium was replaced with a fresh high-glucose media and the cells were incubated for 2 or 24 hours, in $37^{\circ} \mathrm{C}, 5 \% \mathrm{CO}_{2}$. Following 2 or 24 hours of recovery, nuclear DSBs were determined using the Oxiselect DNA Double Stranded Break Staining kit. Nuclei were stained with Hoechst 33342, NucBlue live cell stain (Molecular probes, Life technologies Eugene OR USA). Preparates were examined by fluorescence confocal microscopy with X60 magnification (Nikon A1R) and the amount of nuclei with DSB was estimated by observing at least a hundred nuclei, calculating the relative number of $\mathrm{YH} 2 \mathrm{AX}$ foci per nucleus, using the Image $\mathrm{J}$ software https://imagej.nih.gov/ij/.

For double staining experiments the cells were fixed, permeabilized and blocked (according to the manufacturer's instructions) and cells incubated simultaneously for $1 \mathrm{hr}$ with both antibodies (1:100 for YH2AX , 1:250 for Ki-67 and subsequently incubated with a mix of two secondary antibodies (FITC and Cy5 , respectively) washed and stained with NucBlue.

\section{7 mRNA Expression by Linear Amplification and Sequencing; CEL-Seq}

Total RNA was from extracted (3-5 separate occasions) HFF-shCOX4I1 and control cells HFF-CV. Qualification and quantification were measured using Qbit. Hi-seq assay using CEL-Seq approach was performed at the of the Technion Genome Center, Haifa Israel. Statistic and bioinformatics analyses were performed in collaboration with the Bioinformatics unit of the Hebrew University of Jerusalem, Faculty of Medicine. Differential expression analysis was done with the DESeq2 package (v1.22.1). [26]. In order to 
identify altered biological functions between HFF-shCOX4I1 and HFF), we ran gene set enrichment analysis

(GSEA, reference: https://www.ncbi.nlm.nih.gov/pmc/articles/PMC1239896/). GSEA uses whole differential expression data (cut-off independent) to determine whether a priori-defined sets of genes show statistically significant, concordant differences between two biological states. We used the hallmark gene set collection from the molecular signatures database (MsigDB). The expression data results (GSE166429) are available on the GEO (Gene Expression Omnibus) website (https://www.ncbi.nlm.nih.gov/geo/) (accessed on 20 February 2021).

\subsection{Quantitative reverse transcription polymerase chain reaction (RT-qPCR)}

Total RNA was isolated from patient, HFF-shRNA, HFF-CV and healthy control primary fibroblasts with TriReagent (Telron, Isarel) and cDNA from poly(A)+mRNA was generated using Improm II, Promega, Madison, WI, USA. Real time, quantitative PCR for the quantification of XPC, PCNA, GMPR2, IMPDH2, UPF3B, COX4I1, COX4I2, GUSB and GAPDH transcripts - was performed using Fast SYBR GreenMaster Mix and the ABI PRISM7900HT sequence detection system (Applied Biosystems, Foster City, CA, USA). Primer sequences used for qPCR are supplied the supplementary material.

\subsection{Homologous Recombination Assay}

A homologous recombination assay was performed according to the manufacturer's instructions (Norgen Biotek Corp., cat \#35600). In brief, HEK293 cells expressing either control vector (CV) or shCOX4I1 were co-transfected with both dl-1 and dl-2 plasmids (or negative and positive control plasmids), and total genomic DNA was isolated 24 hours later using DNeasy® Blood\&Tissue Kit (catalog No. 69504 \&69506; Qiagen). PCR reaction was performed using the supplied primers to determine HR efficiency and with the manufacturer's recommendation- 2X PCR Master Mix (Norgen Biotek Cat\# 28007). If HR in COX4-1deficient cells is perturbed, the $\mathrm{dl}-1$ and $\mathrm{dl}-2$ plasmids will less recombine to produce a PCR product than in the control cells; therefore, the amount of PCR product is directly associated with HR efficiency. The amount of recombinant product for each reaction was calculated by comparing the intensity of the HR product to that of the intensities observed in the control (dl-1\&dl-2). Analysis of PCR products was performed using the Image $\mathrm{J}$ software https://imagej.nih.gov/ij/.

\subsection{Statistical analysis}

All experiments were performed in triplicates on at least two different occasions. Statistical analysis was done by two tailed-student's unpaired $t$-test using a IBM SPSS statistics for Windows, version 24.0. software (IBM Corp. Armonk, NY, USA). $p$ values $<0.05$ were considered statistically significant. 


\section{Declarations}

Acknowledgements

This work was supported by the Israeli Science Foundation; ISF (grant \#1059/19). The authors declare no conflict of interest. Tal Katz-Ezov Technion Genome Center Haifa is acknowledged for CEL-Seq analysis. Dr. Sharona Elagvish and Shmuel Ruppo from the Info-CORE, bioinformatics unit of the I-CORE at the Hebrew University of Jerusalem are acknowledged for bioinformatic analysis. Prof. Jacob (Kobi) Rachmilewitz, Hadassah Medcial Center and the Faculty of Medicine, Hebrew University of Jerusalem is acknowledged for advice and fruitful discussions.

\section{Author contributions}

Conceptualization and Investigation, Liza Douiev, and Ann Saada; Funding acquisition, Ann Saada; Methodology and Interpretation; Liza Douiev, Chaya Miller, Shmuel Ruppo, Bassam Abu-Libdeh, Ann Saada; Project administration and Supervision, Ann Saada; Writing original draft, Liza Douiev

\section{Additional Information}

The authors declare no competing interests

\section{References}

1. Sinkler, C. A., Kalpage, H., Shay, J., Lee, I., Malek, M. H., Grossman, L. I., \& Hüttemann, M. Tissue- and Condition-Specific Isoforms of Mammalian Cytochrome $c$ Oxidase Subunits: From Function to Human Disease. Oxid. Med. Cell. Longev. 10.1155/2017/1534056 1534056 (2017).

2. Vogt, S., Ramzan, R., Grossman, L.I., Singh, K.K., Ferguson-Miller, S., Yoshikawa, S., Lee, I., Hüttemann M. Mitochondrial respiration is controlled by Allostery, Subunit Composition and Phosphorylation Sites of Cytochrome c Oxidase: A trailblazer's tale - Bernhard Kadenbach. Mitochondrion. 60,228-233 (2021).

3. Ramzan, R., Kadenbach, B., Vogt, S. Multiple Mechanisms Regulate Eukaryotic Cytochrome C Oxidase. Cells. 10, 10.3390/cells10030514. (2021) 
4. Rak, M., Bénit, P., Chrétien, D., Bouchereau, J., Schiff, M., El-Khoury, R., Tzagoloff, A., Rustin, P. Mitochondrial cytochrome c oxidase deficiency. Clin. Sci. (Lond).130,393-407 (2016).

5. Brischigliaro, M., \& Zeviani, M. (2021). Cytochrome c oxidase deficiency. Biochim Biophys. Acta. Bioenerg. 1862, 10.1016/j.bbabio.2020.148335148335 (2021).

6. Čunátová, K., Reguera, D.P., Houštěk, J., Mráček, T., Pecina, P. Role of cytochrome c oxidase nuclearencoded subunits in health and disease. Physiol. Res. 69, 947-965 (2020)

7. Gattermann, N., Retzlaff, S., Wang, Y.L., Hofhaus, G., Heinisch, J., Aul, C., Schneider, W. Heteroplasmic point mutations of mitochondrial DNA affecting subunit I of cytochrome $c$ oxidase in two patients with acquired idiopathic sideroblastic anemia. Blood. 90, 4961-72 (1997).

8. Shteyer, E., Saada, A., Shaag, A., Al-Hijawi, F.A., Kidess, R., Revel-Vilk, S., Elpeleg, O. Exocrine pancreatic insufficiency, dyserythropoeitic anemia, and calvarial hyperostosis are caused by a mutation in the COX4I2 gene. Am J Hum Genet. 84,412-417 (2009).

9. Abu-Libdeh, B., Douiev, L., Amro, S., Shahrour, M., Ta-Shma, A., Miller, C., Elpeleg, O. \& Saada, A. Mutation in the COX4I1 gene is associated with short stature, poor weight gain and increased chromosomal breaks, simulating Fanconi anemia. Eur. J. Hum. Genet. 25, 1142-1146 (2017).

10. Douiev, L., Miller, C., Ruppo, S., Benyamini, H., Abu-Libdeh, B., \& Saada, A. Upregulation of COX4-2 via HIF-1a in Mitochondrial COX4-1Deficiency. Cells. 10.3390/cells10020452 (2021).

11. Douiev, L., Abu-Libdeh, B., \& Saada, A. Cytochrome c oxidase deficiency, oxidative stress, possible antioxidant therapy and link to nuclear DNA damage. Eur.J.Hum.Gen. 26,579-581 (2018).

12. Douiev, L., \& Saada, A. The pathomechanism of cytochrome c oxidase deficiency includes nuclear DNA damage. Biochim. Biophys. Acta. Bioenerg. 1859, 893-900 (2018).

13. Pagano, G., Talamanca, A. A., Castello, G., d'Ischia, M., Pallardó, F. V., Petrović, S., Porto, B., Tiano, L., \& Zatterale, A. Bone marrow cell transcripts from Fanconi anaemia patients reveal in vivo alterations in mitochondrial, redox and DNA repair pathways. Eur. J. Haematol. 91, 141-151 (2013).

14. Ravera, S., Vaccaro, D., Cuccarolo, P., Columbaro, M., Capanni, C., Bartolucci, M., Panfoli, I., Morelli, A., Dufour, C., Cappelli, E., \& Degan, P. Mitochondrial respiratory chain Complex I defects in Fanconi anemia complementation group A. Biochimie. 95, 1828-1837 (2013).

15. Cappelli, E., Cuccarolo, P., Stroppiana, G., Miano, M., Bottega, R., Cossu, V., Degan, P., \& Ravera, S. Defects in mitochondrial energetic function compels Fanconi Anaemia cells to glycolytic metabolism. Biochim. Biophys. Acta. Mol. Basis Dis.1863, 1214-1221 (2017).

16. Yi, T., Feng, Y., Sundaram, R., Tie, Y., Zheng, H., Qian, Y., You, D., Yi, T., Wang, P., \& Zhao, X. Antitumor efficacy of PARP inhibitors in homologous recombination deficient carcinomas. Int. J. Cancer. 145,1209-1220 (2019).

17. Debacq-Chainiaux F., Ben Ameur R., Bauwens E., Dumortier E., Toutfaire M. \& Toussaint O. StressInduced (Premature) Senescence. 242-263 In: Rattan S., Hayflick L. (eds) Cellular Ageing and Replicative Senescence. Healthy Ageing and Longevity.Springer, Cham (2016).

18. Kogot-Levin, A., Saada, A., Leibowitz, G., Soiferman, D., Douiev, L., Raz, I., Weksler-Zangen, S. Upregulation of Mitochondrial Content in Cytochrome c Oxidase Deficient Fibroblasts. PLoS One. 
11.1371/journal.pone.0165417 (2016).

19. Wiley, C. D., \& Campisi, J. From Ancient Pathways to Aging Cells-Connecting Metabolism and Cellular Senescence. Cell metabolism. 23, 1013-1021 (2016).

20. Sulli, G., Di Micco, R., \& d'Adda di Fagagna, F. Crosstalk between chromatin state and DNA damage response in cellular senescence and cancer. Nat. Rev. Cancer. 12,709-720 (2012).

21. Bourseguin, J., Bonet, C., Renaud, E., Pandiani, C., Boncompagni, M., Giuliano, S., Pawlikowska, P., Karmous-Benailly, H., Ballotti, R., Rosselli, F., \& Bertolotto, C. FANCD2 functions as a critical factor downstream of MiTF to maintain the proliferation and survival of melanoma cells. Sci Rep. 6, 10.1038/srep36539 (2016).

22. Helbling-Leclerc, A., Garcin, C., \& Rosselli, F. Beyond DNA repair and chromosome instability-Fanconi anaemia as a cellular senescence-associated syndrome. Cell. Death. Differ.28,1159-1173 (2021).

23. Shah, A., George, M., Dhangar, S., Rajendran, A., Mohan, S., Vundinti, BR. Severe telomere shortening in Fanconi anemia complementation group L. Mol.Biol. Rep.48,585-593 (2021).

24. Kubben, N., Misteli, T. Shared molecular and cellular mechanisms of premature ageing and ageingassociated diseases. Nat Rev Mol Cell Biol. 18,595-609 (2017).

25. Douiev, L., Soiferman, D., Alban, C., Saada, A. The Effects of Ascorbate, N-Acetylcysteine, and Resveratrol on Fibroblasts from Patients with Mitochondrial Disorders. J. Clin. Med.6, 10.3390/jcm6010001 (2016).

26. Subramanian, A., Tamayo, P., Mootha, V. K., Mukherjee, S., Ebert, B. L., Gillette, M. A., Paulovich, A., Pomeroy, S. L., Golub, T. R., Lander, E. S., \& Mesirov, J. P. Gene set enrichment analysis: a knowledgebased approach for interpreting genome-wide expression profiles. Proc. Natl. Acad. Sci. U S A, 102 (2005).

\section{Figures}




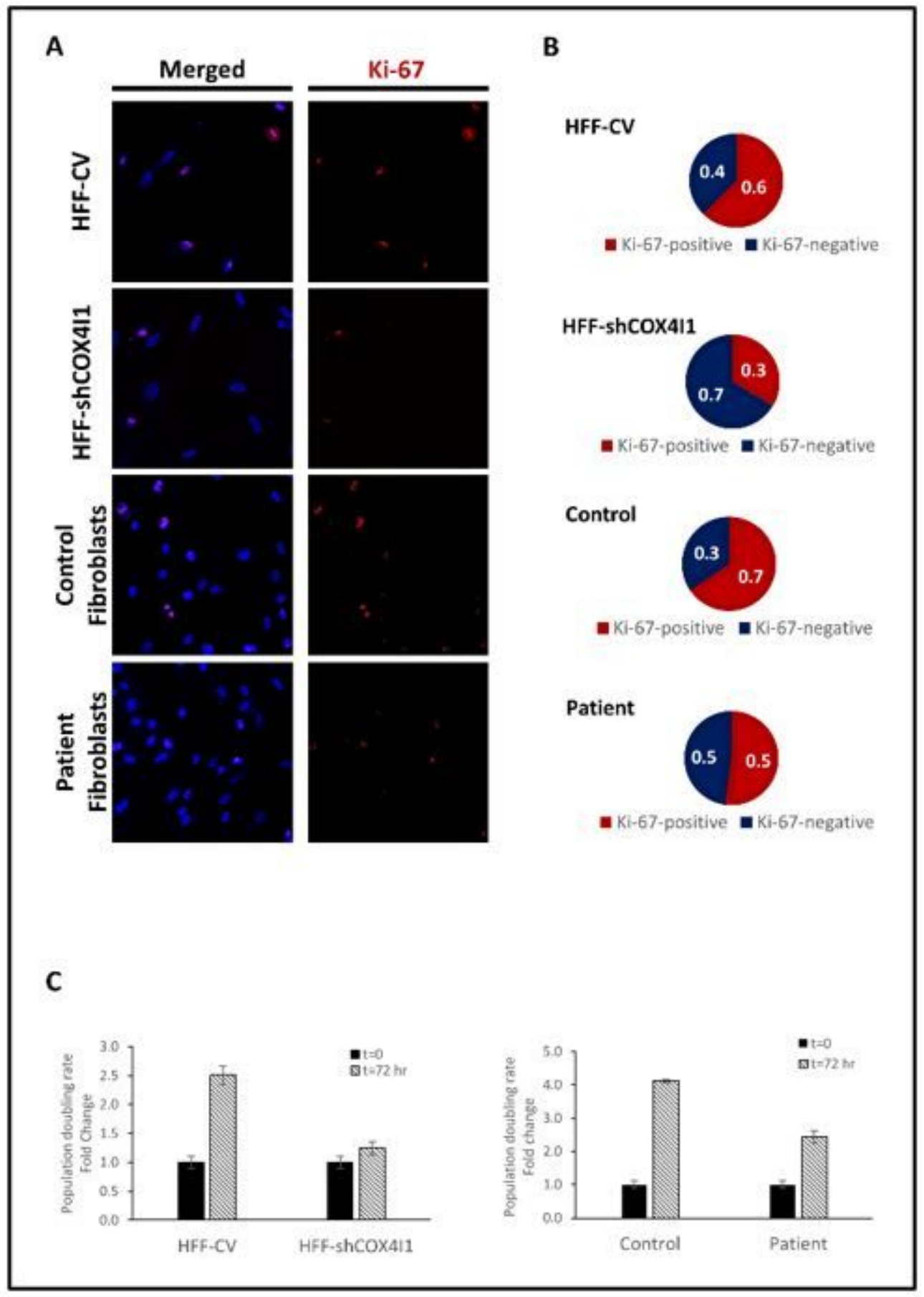

Figure 1

Decreased proliferation in COX4-1-deficient cells. Both COX4-1-deficient cells (HFF-shCOX4I1 and patient) and their corresponding controls (HFF-CV and healthy control), from similar passages were seeded in equal numbers in triplicates $(t=0)$, grown for 72 hours ( $t=72)$ and subsequently stained with Ki-67 antibodies and NucBlue to visualize proliferating cells and nuclei (representative micrograph) (A) which were counted ( $n=$ at least 50 cells for each triplicate) and depicted in pie charts (B) or stained with trypan blue, counted and depicted in bar graphs (mean \pm SEM $n=3$ ). 


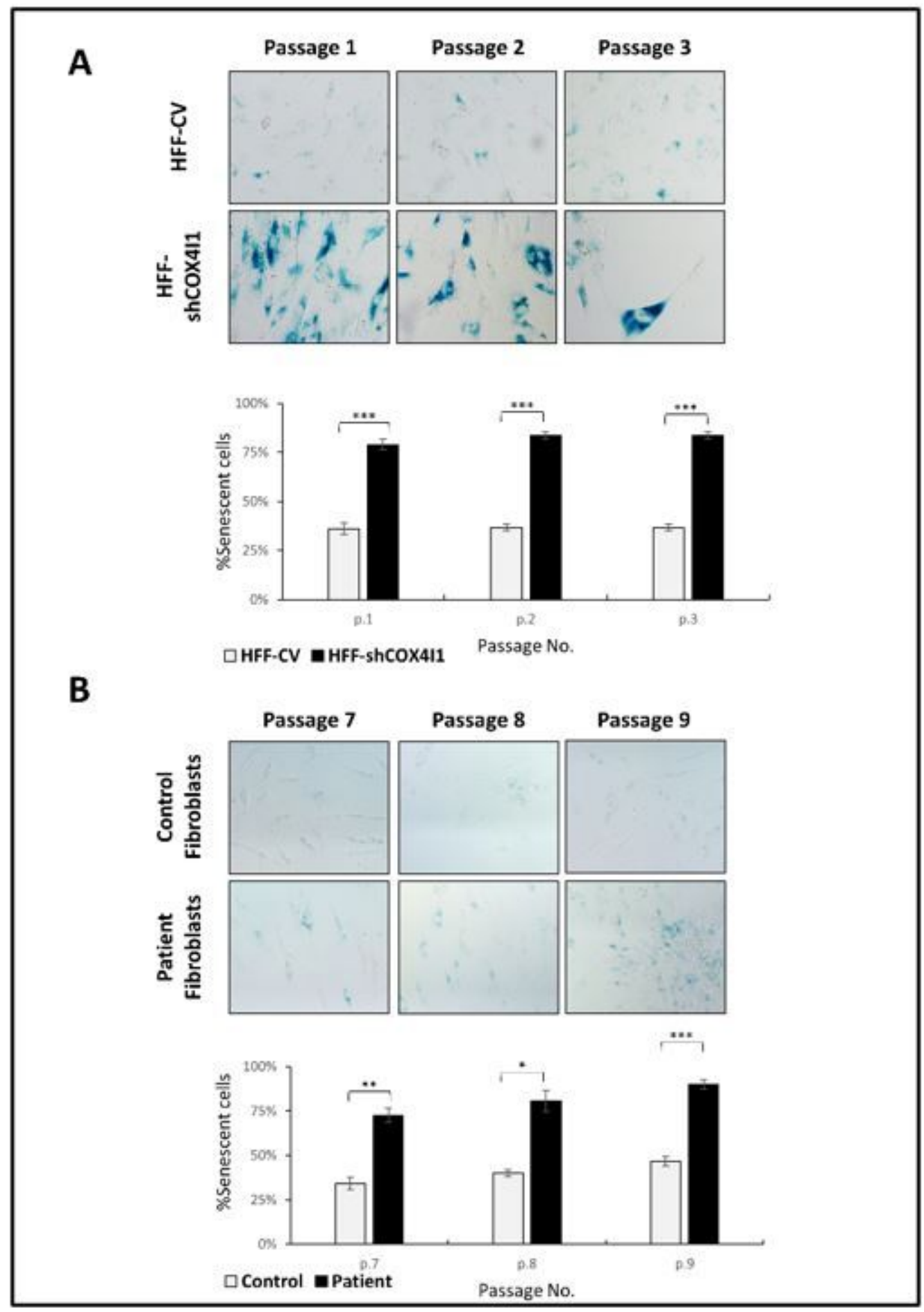

Figure 2

COX4-1 deficiency results in premature cellular senescence. Cellular senescence was detected by staining for senescence-associated $\beta$-galactosidase (SA- $\beta$-gal) staining of both COX4-1-deficient cells (HFFshCOX4I1 (A) and patient (B)) and their corresponding controls (HFF-CV and healthy control). Representative micrographs from three shown constitutive passages $(p)$ are shown $(A, B)$. The percentage of positive (blue) cells was quantified and depicted in bar graphs under the micrographs, (mean \pm SEM $\left.n>100,{ }^{*} p<0.05,{ }^{\star *} p<0.01,{ }^{\star \star *} \mathrm{p}<0.005\right)(A, B)$. 


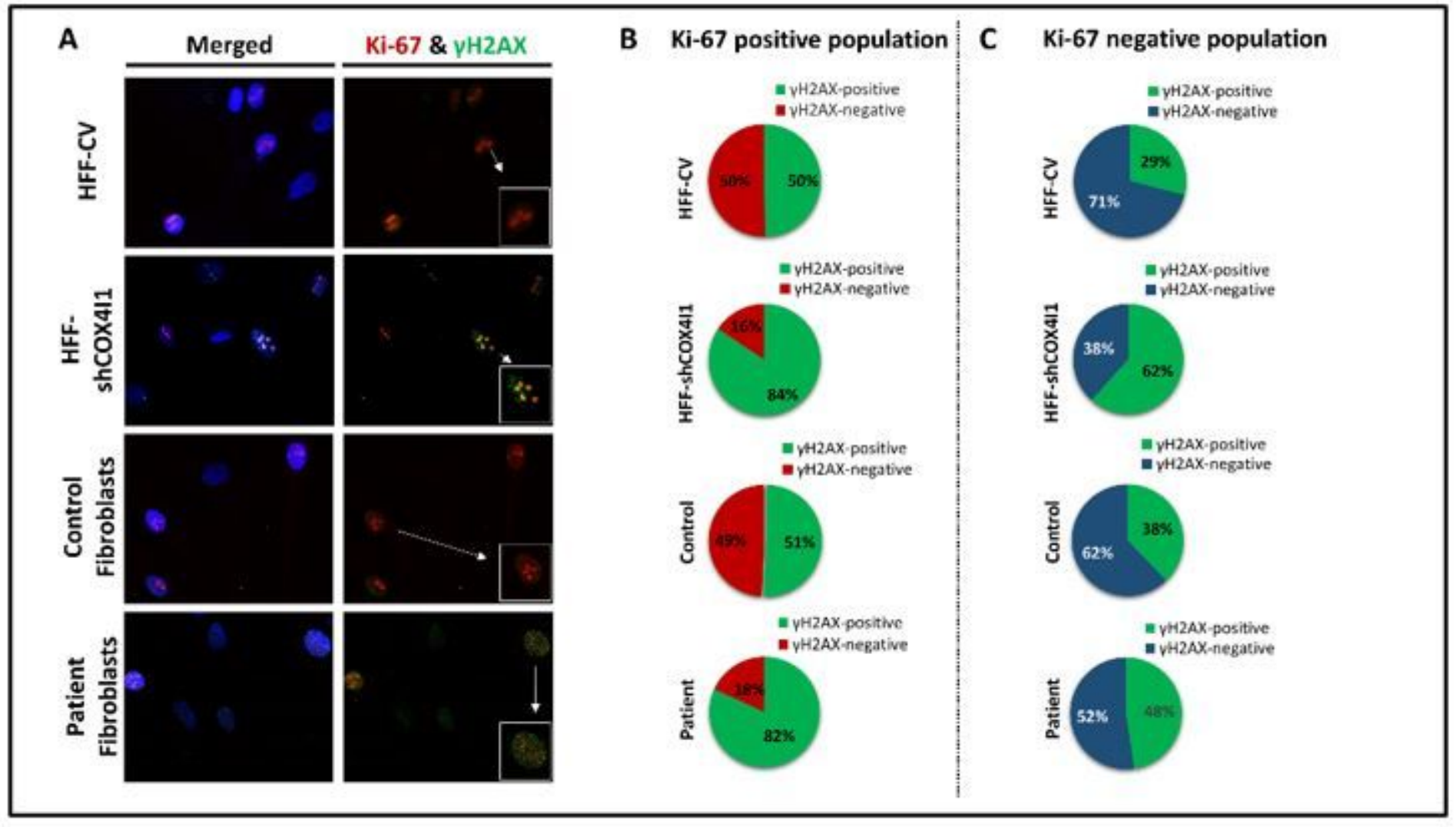

\section{Figure 3}

Elevated levels of DSB coincides with proliferation in COX4-1-deficient cells. Both COX4-1-deficient cells (HFF-shCOX4I1 and patient) and their corresponding controls (HFF-CV and healthy control), from similar passages were seeded in equal amounts overnight on tissue culture slides, were fixed and stained simultaneously for $\mathrm{YH} 2 \mathrm{AX}$ (green) and Ki-67 (red). Nuclei were visualized by Hoechst-3334 (blue) (A). The pie charts represent the distribution of DSBs ( $\mathrm{HH} 2 \mathrm{AX}$, green) in proliferative (Ki-67 positive, B) and in nonproliferative cell (Ki-67 negative, C). At least 100 nuclei of each group of cells were analyzed and quantified. Increased colocalization of $\mathrm{Ki}-67$ and $\mathrm{YH} 2 \mathrm{AX}$ was observed in both COX4-1-deficient cells relative to corresponding controls. 


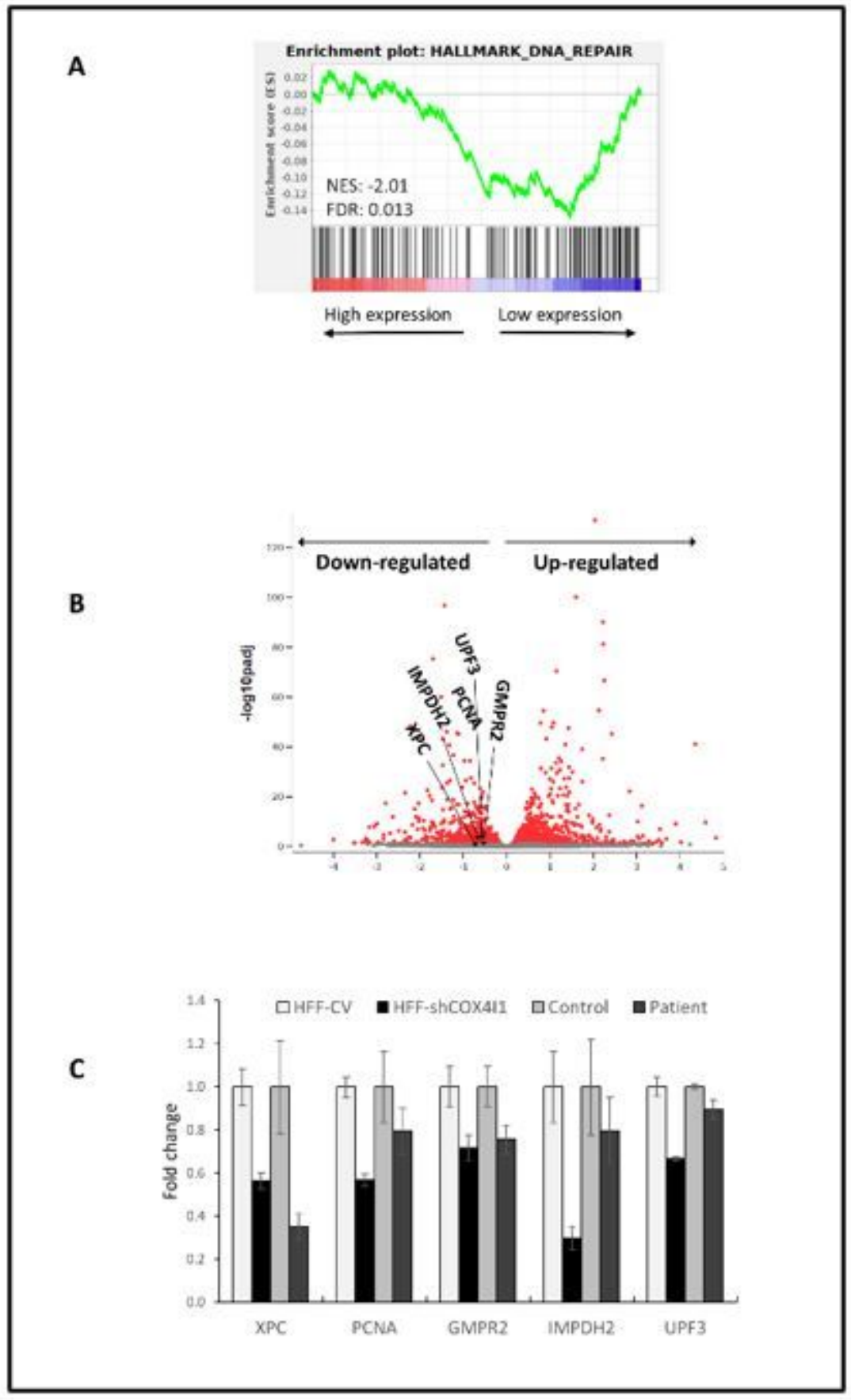

Figure 4

CEL-Seq2 analysis identified DNA repair as one of the top downregulated pathways in COX4-1-deficient cells. 


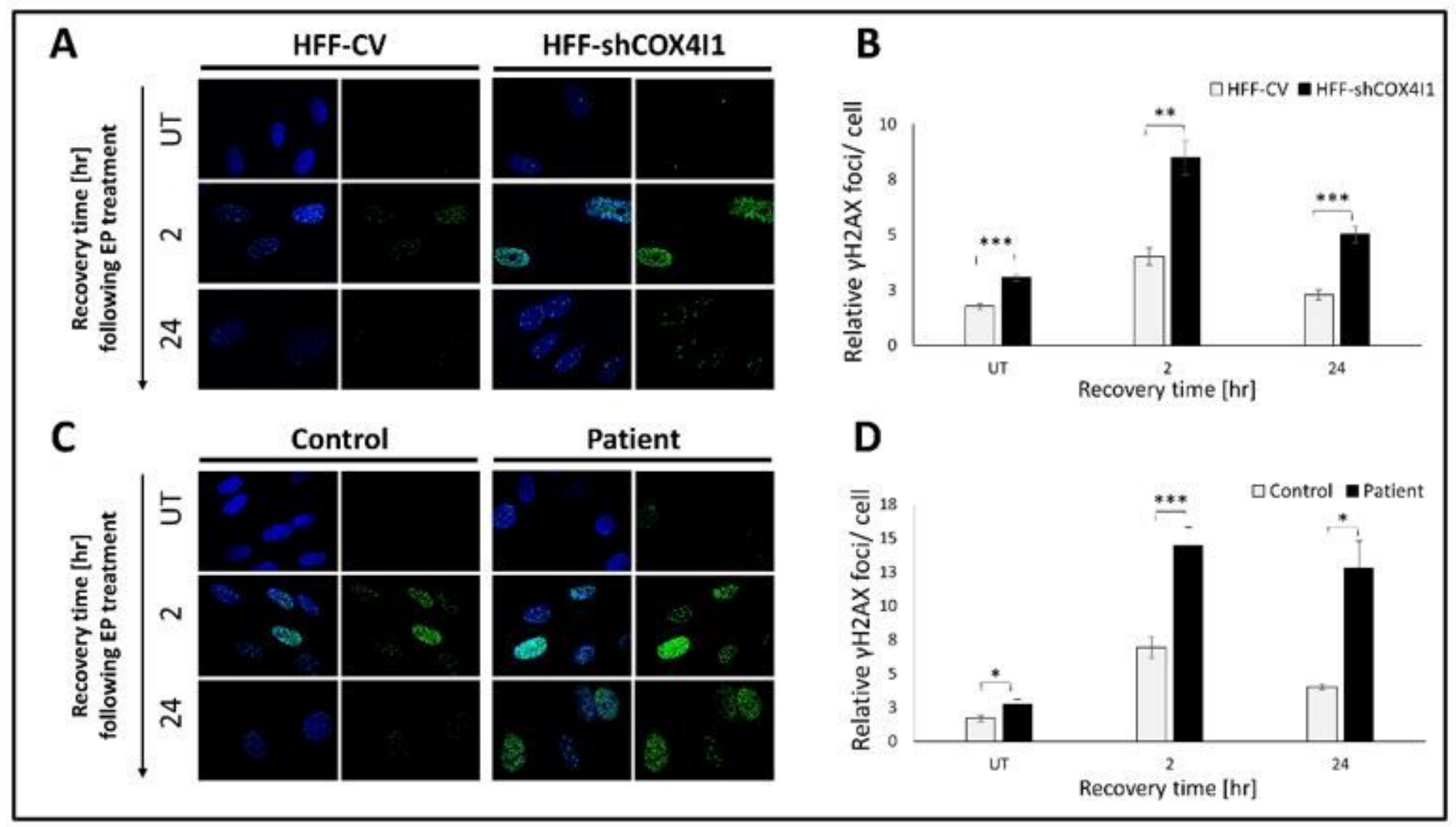

\section{Figure 5}

DNA damage recovery following Etoposide (EP) treatment is less efficient in COX4-1-deficient cells. Both COX4-1-deficient cells (HFF-shCOX4I1 and patient) and their corresponding controls (HFF-CV and healthy control), from similar passages were seeded in equal numbers overnight on tissue culture slides and the following day exposed for 1 hour to $100 \mu \mathrm{M}$ Etoposide; EP. Following 2 or 24 hours of recovery, the cells were stained for DSBs anti-yH2AX antibody (green). Nuclei were visualized by Hoechst-3334 (blue) and the quantity of DSB foci per nucleus was analyzed and compared to untreated cells (UT) and respective controls. The micrographs were quantified and depicted as histograms (B\&D) that the $\mathrm{H} 2 \mathrm{AX}$ foci number per nucleus relative to $t=0$ (meaning, untreated cells) \pm SEM of at least 100 nuclei ${ }^{\star \star \star} p<0.001$. The results suggest that both COX4I1-deficient cells show impaired ability to respond and thereby to recover from DNA damage, while the controls exhibit almost a complete recovery 24 hours after the exposure. 


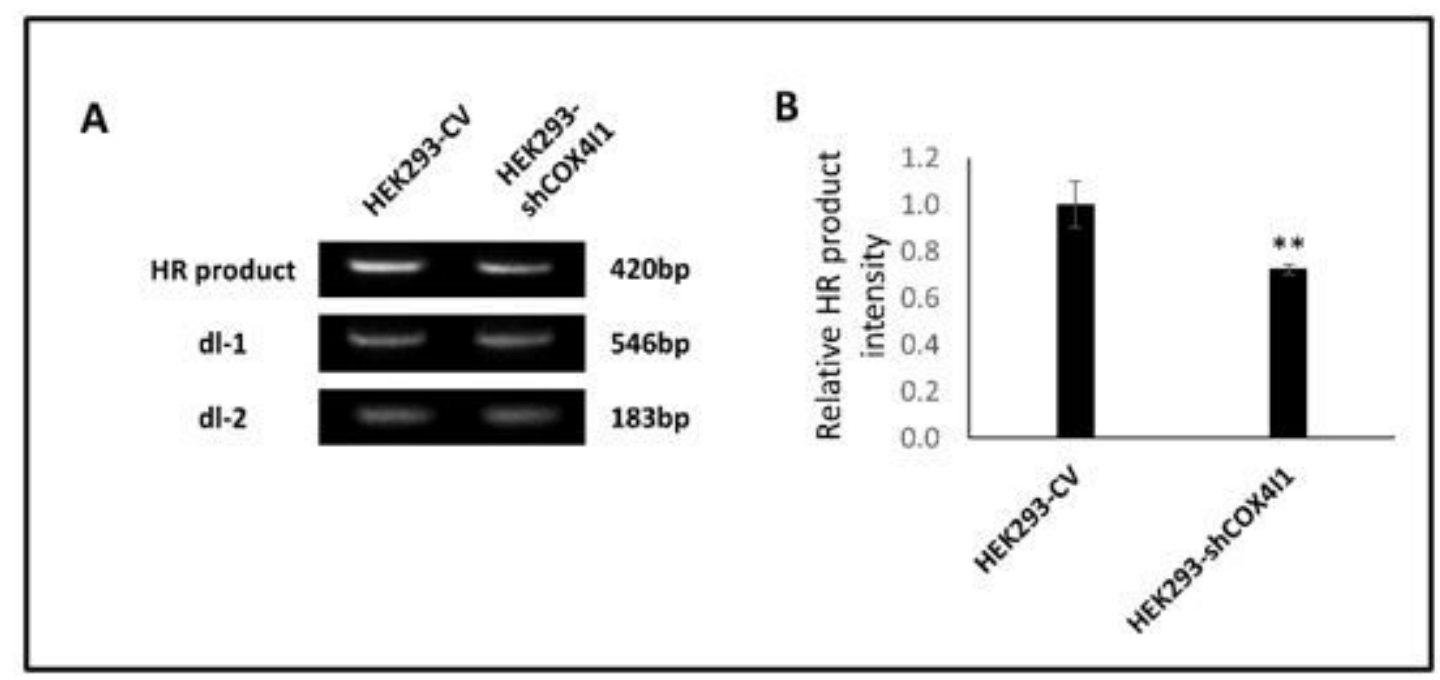

Figure 6

Down-regulation of COX4-1 interferes with homologous recombination (HR) repair. Homologous recombination (HR) assay was performed on HEK293 cell line with deither down-regulation of COX4I1 (HEK293-shCOX4I1) or control HEK293-CV by transfecting with two mutated assay plasmids (dl-1\&dl-2) according to the manufacturer's instructions. DNA was isolated 24 hours later and PCR reaction was performed determine HR efficiency. A weaker intensity of the HR product observed in the HEK293shCOX4I1 relative to that of the HEK293-CV (A, depicting a representative experiment out of three). When normalizing the intensities of the HR product to that of the controls of each type of cell (dl-1\&dl-2), a significant decreased HR product is observed in the COX4-1-deficient cells, relative to that of the corresponding control (C). Values are normalized to the mean value of the control $\pm S E M ; * \star p<0.01 . n=3$

\section{Supplementary Files}

This is a list of supplementary files associated with this preprint. Click to download.

- Supplementarymaterial.docx 